\section{Étienne Marbaix Isabelle Kokorine Jacques Donnez Pierre J. Courtoy Yves Eeckhout}

\title{
Collagénases et métalloprotéases apparentées: un rôle essentiel dans le déclenchement de la menstruation?
}

Deux ensembles d'arguments expérimentaux directs plaident en faveur d'un rôle des métalloprotéinases matricielles dans la dégradation de la matrice extracellulaire de l'endomètre et le déclenchement de la menstruation. L'expression de la collagénase interstitielle, des stromélysines, de la gélatinase $B$ est restreinte à la période péri-menstruelle; la culture d'explants d'endomètres prélevés en dehors de la période menstruelle montre que l'expression, la sécrétion et l'activation des métalloprotéinases matricielles sont réprimées par les stéroïdes sexuels ajoutés à des concentrations physiologiques et déréprimées en leur absence. On conclut que l'activité des ces protéases est libérée juste avant et pendant la menstruation, à la suite de la chute des stéroïdes sexuels, ce qui entraîne la dissolution tissulaire. La compréhension de ces phénomènes devrait mener à une approche thérapeutique nouvelle des méno- et métrorragies.

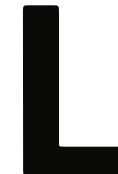

'endomètre, cette muqueuse de quelques millimètres d'épaisseur qui tapisse la cavité utérine, a pour rôle principal d'accueillir l'œuf fécondé. En l'absence de fécondation et de nidation, la chute de la concentration plasmatique des stéroïdes sexuels (progestérone et œstradiol) déclenche une résorption de la matrice extracellulaire associée à des hémorragies intratissulaires qui aboutissent tous les 28 jours environ à la menstruation, c'est-à-dire à l'élimination par voie vaginale de sang contenant des fragments d'endomètre.
Malgré l'importance du processus menstruel en physiologie et en pathologie, et contrastant avec la bonne connaissance de sa régulation hormonale, les mécanismes biochimiques et cellulaires de la menstruation n'ont fait l'objet que de quelques études et sont encore mal compris. Les hypothèses et observations résumées ci-après résultent d'une étroite collaboration entre les auteurs qui pratiquent des disciplines aussi diverses que la gynécologie, l'anatomie pathologique, la biologie cellulaire, la biologie moléculaire et la biochimie. 


\section{RÉFÉRENCES}

1. Ferenczy A. Anatomy and histology of the uterine corpus. In: Kurman RJ, ed. Blaustein's pathology of the female genital tract. New York: Springer-Verlag, 1994: 327-66.

2. More IAR. The normal human endometrium. In: Fox H, ed. Haines and Taylor osietrical and gyncecological pathology. Edinburgh Churchill Livingstone, 1987: vol. 1, 302-19.

3. Christiaens GCML, Sixma IJ, Haspels AA Morphology of haemostasis in menstrual endometrium. Br J Obstet Gnnaecol 1980;87: 425-39.

4. Kruitwagen RFPM. Retrograde menstruation. In: Brosens I, Donnez J, eds. The current status of endometriosis. Research and management. Carnforth: The Parthenon Publishing Group, 1993: 3-17.

5. Aplin JD, Charlton AK, Ayad SD. An immunohistochemical study of human endometrial extracellular matrix during the menstrual cycle and first trimester of pre gnancy. Cell 'Tissue Res 1988; 253: 231-40.

6. Woessner JF. Uterus, cervix and ovary. In: Weiss JB, Jayson MIV, eds. Collagen in health and disease. Edinburgh: Churchill Livingstone, 1982: 506-27.

7. Markee JE. Menstruation in intraocular endometrial transplants in the Rhesus monkey. Contributions to Embryology 1940; 28 : 219-308.

8. Henzl MR, Smith RE, Boost G, Tyler ET Lysosomal concept of menstrual bleeding in humans. J Clin Endocr 1972; 34 : 860-75.

9. Cornillie F, Brosens I, Belsey EM, Marbaix E, Baudhuin P, Courtoy PJ. Lysosomal enzymes in the human endometrium : a biochemical study in untreated and levonorgestrel-treated women. Contraception 1991 43: $387-400$.

10. Downie J, Poyser NL, Wunderlich M. Levels of prostaglandins in human endome trium during the normal menstrual cycle. $J$ Physiol 1974; 236: 465-72.

11. Eeckhout Y. Tissue breakdown. In : D'Arcangues C, Fraser IS, Newton JR, Odlind V, eds. Contraception and mechanisms of endometrial bleeding. Cambridge: Cambridge University Press, 1990: 431-9.

12. Birkedal-Hansen $\mathrm{H}$, Moore WGI, Bodden MK, Windsor LJ, Birkedal-Hansen B DeCarlo A, Engler JA. Matrix metalloproteinases: a review. Crit Rew Oral Biol Med 1993. 4: $197-250$.

\section{Les modifications de l'endomètre au cours du cycle menstruel}

L'endomètre est constitué de deux couches. La couche basale, qui atteint à peine $1,5 \mathrm{~mm}$ d'épaisseur et ne varie guère au cours de la vie reproductrice, est aussi appelée résiduelle car elle persiste lors des menstruations. La couche superficielle est appelée fonctionnelle car elle subit des modifications importantes au cours de chaque cycle menstruel (figure $1 A,(;, E)[1]$. Pendant la phase préovulatoire, les cellules épithéliales glandulaires, les cellules stromales et les cellules endothéliales prolifèrent à partir de la couche basale sous l'influence de l'œstradiol, ce qui reconstitue en une dizaine de jours une muqueuse de 4 à $5 \mathrm{~mm}$ d'épaisseur. Après l'ovulation, une différenciation sécrétoire apparaît sous l'influence de la progestérone, d'abord visible dans l'épithélium glandulaire sous forme de dépôts de glycogène et de glycoprotéines, puis dans la lumière des glandes. Après cette phase sécrétoire précoce, le stroma endométrial se distend davantage par œdème, attribué à une transsudation induite par le second pic d'œstradiol plasmatique. Au moment présumé de l'implantation de l'œuf fécondé, l'endomètre atteint 5 à $6 \mathrm{~mm}$ d'épaisseur.

Par la suite, l'œdème régresse tandis que les cellules stromales prolifèrent à nouveau, grossissent en se chargeant de glycogène, de mucopolysaccharides et de lipides, et sécrètent des constituants des lames basales. On les appelle cellules prédéciduales car elles sont les précurseurs des cellules déciduales qui peuplent la décidua ou caduque. Ce terme désigne l'endomètre gestationnel qui sera éliminé immédiatement après l'accouchement. Les cellules prédéciduales apparaissent d'abord sous l'épithélium de surface et autour des artérioles spiralées, endroits qui seront infiltrés préférentiellement par les cellules trophoblastiques intermédiaires, issues du produit de conception après son implantation. Les artérioles spiralées, caractéristiques de la femme et des primates qui présentent une menstruation régulière [2], s'allongent de manière disproportionnée par rapport à l'épaisseur de l'endomètre. A la fin de la phase sécrétoire, les glandes se festonnent davantage, la prédécidualisation s'étend et des cellules lymphoïdes granuleuses impliquées dans le contrôle immunitaire de la grossesse infiltrent le stroma endométrial.

En l'absence de grossesse, la menstruation survient environ 28 jours après le début de la période menstruelle précédente. Elle dure 4 à 5 jours et se caractérise par une réduction considérable de l'épaisseur de l'endomètre, pratiquement ramené à la seule couche basale. La menstruation est précédée d'un afflux de polynucléaires neutrophiles, de lésions endothéliales focales et de foyers d'hémorragie dans la muqueuse. Pendant les deux premiers jours des menstrues, les portions superficielles de la muqueuse s'effondrent et se délitent par endroits à la suite d'une rétraction du stroma, des thrombi de fibrine apparaissent dans les lumières vasculaires et dans le stroma, et des fragments de muqueuse altérée sont libérés dans la cavité endométriale en même temps que du sang incoagulable [3]. ('es fragments tissulaires ne sont pas nécrosés puisqu'on peut les cultiver. Leur reflux par les trompes utérines dans la cavité péritonéale est fréquemment observé et a été impliqué dans la pathogenèse de l'endométriose du petit bassin [4]. La composition biochimique de la matrice extracellulaire de l'endomètre fonctionnel varie au cours du cycle menstruel [5]. Les collagènes de types I, III et $\mathrm{V}$ sont abondants tout au long des phases proliférative et sécrétoire, réalisant un réseau de fibres réticuliniques bien identifiable par imprégnation argentique (figure $1 B, I)$ [ $[6]$. En revanche, le collagène de type VI disparaît du stroma endométrial au cours de la phase sécrétoire, sauf dans les parois vasculaires et autour des glandes. Ce type de collagène est impliqué dans la formation d'un réseau microfibrillaire et sa disparition favoriserait une expansion de l'interstitium endométrial. Durant la fin de la phase sécrétoire, le stroma s'enrichit essentiellement de laminine, de protéoglycannes riches en héparanne-sulfate, de collagène de type IV et de fibronectine, tous constituants des lames basales sécrétés par les cellules stromales. Juste avant la menstruation, la rétraction 

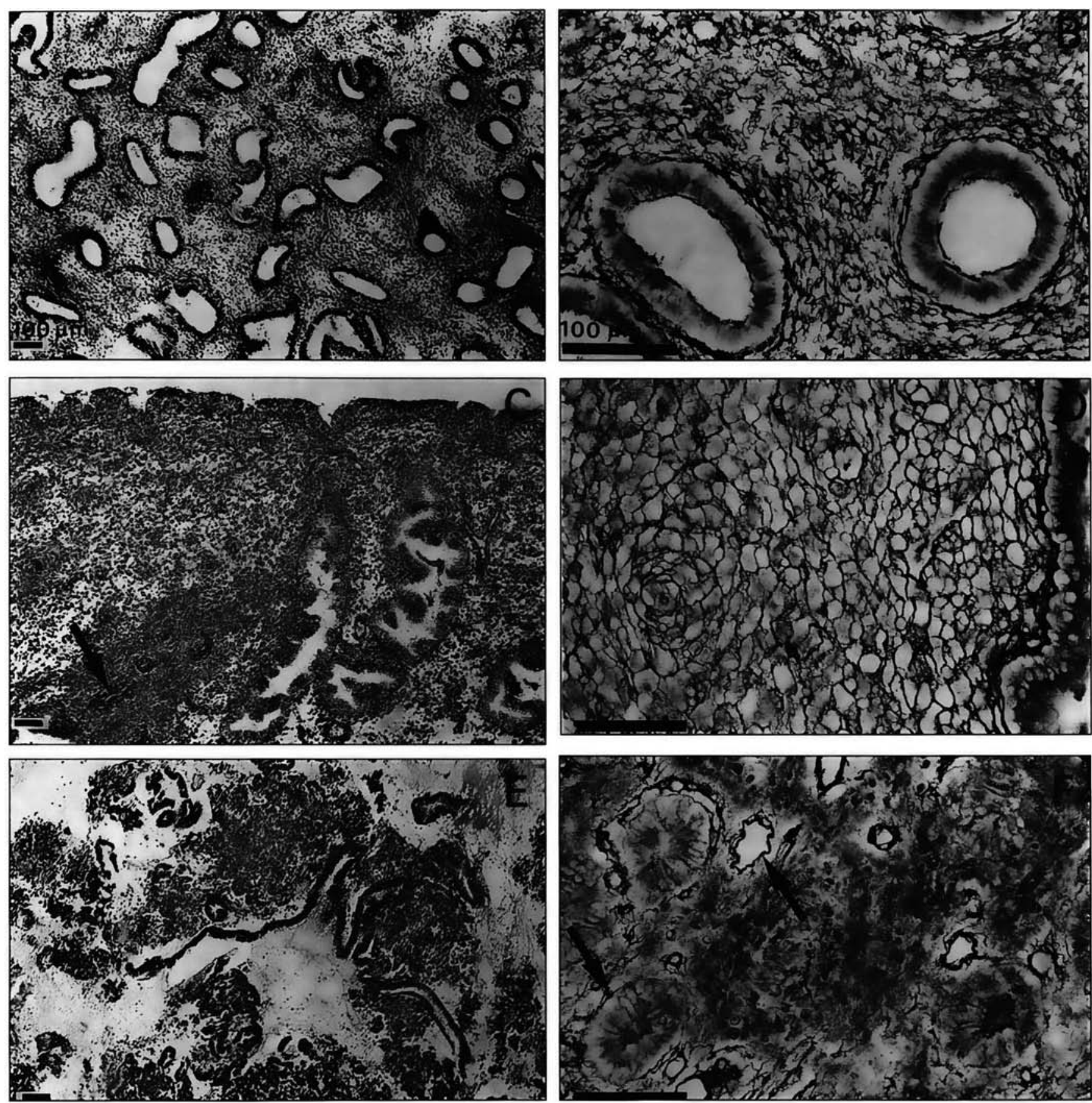

Figure 1. Organisation de la matrice extracellulaire de l'endomètre au cours du cycle menstruel. Les microphotographies de gauche sont des vues d'ensemble de sections histologiques colorées à l'hémalun et à l'éosine et celles de droite intéressent les mêmes tissus à plus fort grossissement, après imprégnation argentique des fibres réticuliniques. Les barres correspondent à $100 \mathrm{\mu m}$. A, B. Endomètre en début de phase sécrétoire. Le stroma est œedémateux et les fibres réticuliniques, abondantes, lui confèrent un aspect chevelu. $C, D$. Endomètre en fin de phase sécrétoire. Les cellules prédéciduales forment un manchon autour d'une artériole spiralée (C, flèche); ailleurs, le stroma est œdémateux et infiltré de nombreuses cellules lymphoïdes. Dans cette portion superficielle de la muqueuse (D), les fibres réticuliniques dessinent un maillage autour des cellules prédéciduales. $\boldsymbol{E}, \boldsymbol{F}$. Endomètre menstruel. La muqueuse se désagrège, les glandes se ratatinent et le stroma se délite par manque d'armature conjonctive, comme en atteste la disparition des fibres réticuliniques, hormis autour de certains vaisseaux et de rares segments glandulaires ( $F$, flèches). 


\section{RÉFÉRENCES}

13. Sato H, Takino T, Okada Y, Cao J, Shinagawa A, Yamamoto E, Seiki M. A matrix metalloproteinase expressed on the surface of invasive tumour cells. Nature 1994;370: $61-5$.

14. Wolf $C$, Lefebvre $O$, Rouyer N, Chenard M, Bellocq J, Rio M, Chambon P, Basset P. Protéases d'origine stromale et progression tumorale. médecine/sciences 1994; 10:507-15.

15. Murphy GJP, Murphy G, Reynolds IJ The origin of matrix metalloproteinases and their familial relationships. FEBS Let $1991 ; 289: 4-7$.

16. Freije JMP, Diez-Itza I, Balbin M, Sanchez LM, Blasco R, Tolivia J, López-Otin C Molecular cloning and expression of collagenase-3, a novel human matrix metalloproteinase produced by breast carcinomas. $J$ Biol Chem $1994 ; 269$ : 16766-73.

17. Eeckhout Y, Vaes G. Further studies on the activation of procollagenase, the laten precursor of bone collagenase. Effects of ysosomal cathepsin B, plasmin and kallikrein, and spontaneous activation. Biochem $1977 ; 166: 21-31$

18. Vassali JD, Sappino AP, Belin D. The plasminogen activator/plasmin system. Clin Invest $1991 ; 88$ : 1067-72.

19. Rybo G. Plasminogen activators in the endometrium. II. Clinical aspects. Variation in the concentration of plasminogen activators during the menstrual cycle and its relation to menstrual blood loss. Acta Obste Gynecol Scand 1966; 45: 429-50.

20. Casslén B, Astedt B. Occurrence of both urokinase and tissue plasminogen activator in the human endometrium. Contraception $1983 ; 28: 553-64$.

21. Plow EF, Freaney DE, Plescia J, Mile LA. The plasminogen system and cell surfaces: evidence for plasminogen and urokinase receptors on the same cell type. $J$ Cell Biol $1986 ; 103$ : 2411-20

22. Marbaix E, Kokorine I, Henriet P, Donnez J, Courtoy PJ, Eeckhout Y. The expression of interstitial collagenase in human endometrium is controlled by progesterone and by estradiol and is related to menstruation. Biochem J 1995; 305: 1027-30.

23. Hampton AL, Salamonsen IA. Expression of messenger ribonucleic acid encoding matrix metalloproteinases and their tissue inhibitors is related to menstruation Endocrinol 1994 ; 141 : R1-3.

24. Rodgers WH, Matrisian LM, Giudice LC Dsupin B, Cannon P, Svitek C, Gorstein F, Osteen KG. Patterns of matrix metalloproteinase expression in cycling endometrium imply differential functions and regulation by steroid hormones. J Clin Invest 1994; 94 . du stroma et l'effritement du réseau réticulinique observés en microscopie optique (figure $1 F$ ) sont le reflet d'une dégradation de l'armature conjonctive de la muqueuse [6].

\section{Interprétations classiques de la menstruation}

La menstruation a été minutieusement étudiée pour la première fois par J.E. Markee, grâce à l'implantation d'endomètre de singes Rhésus dans la chambre antérieure de leur œil [7]. Dans son étude, les événements constants sont la rétraction tissulaire et la stase sanguine, attribuée à un tassement des artérioles spiralées, dans les 5 jours qui précèdent la menstruation, puis une vasoconstriction intense de ces artérioles dans les 24 heures qui précèdent les hémorragies. En particulier, le volume des implants endométriaux peut diminuer de près de dix fois avant les hémorragies menstruelles ! Cette régression tissulaire n'est pas toujours suivie d'hémorragie et l'on observe d'ailleurs un amincissement de la muqueuse endométriale sans hémorragie au cours du cycle reproducteur de mammifères non primates. En outre, dans le système expérimental de Markee, des petits fragments tissulaires se détachent des implants au cours de la menstruation et se désagrègent rapidement. La régression de la muqueuse endométriale, la libération de fragments tissulaires et leur dissolution indiquent l'existence d'un processus de digestion tissulaire durant la période périmenstruelle, c'est-à-dire immédiatement avant et pendant la menstruation.

Cette digestion tissulaire a d'abord été imputée aux hydrolases lysosomiales [8], dont l'activité spécifique est particulièrement élevée dans l'endomètre [9]. Des études morphologiques démontrent en effet une autophagie accrue en fin de phase sécrétoire et suggèrent une sécrétion d'enzymes lysosomiales dans la matrice extracellulaire en milieu et fin de phase sécrétoire [8]. L'augmentation de la production des prostaglandines $\mathrm{F}_{2 \alpha}$ en phase sécrétoire et $\mathrm{E}_{2}$ au moment de la menstruation [10] pourrait provoquer une vasoconstriction veineuse aboutissant à la stase sanguine et être, directement ou indirectement, responsable de souffrance des cellules endothéliales, ce qui conduirait à la libération de leurs enzymes lysosomiales.

La sécrétion d'une enzyme lysosomiale a bien été démontrée lors de cultures d'explants endométriaux [9]. Cependant, la concentration tissulaire des enzymes lysosomiales n'augmente pas en fin de phase sécrétoire. De plus, aucune corrélation n'a pu être établie entre les saignements anormaux de patientes traitées par un progestatif de synthèse et l'abondance d'enzymes lysosomiales dans leur endomètre ou l'importance de la sécrétion de ces enzymes en culture organotypique [9]. Pour cette raison, notre équipe s'est orientée vers d'autres enzymes susceptibles d'intervenir dans la dégradation de la matrice extracellulaire [11].

\section{Les métalloprotéinases matricielles et leurs activateurs}

Les métalloprotéinases matricielles (MMP) constituent une famille d'enzymes capables d'hydrolyser les protéines de la matrice extracellulaire à $\mathrm{pH}$ neutre [12]. Les MMP sont impliquées dans le remodelage de la matrice extracellulaire qui accompagne de nombreux processus normaux et pathologiques. Ces protéases sont synthétisées sous forme de proenzymes inactives. A l'exception d'une MMP de type membranaire [13] et de la collagénase des granules spécifiques des neutrophiles [12], les MMP sont sécrétées immédiatement après leur synthèse et activées par clivage protéolytique d'un propeptide $\mathrm{N}$-terminal d'environ $10 \mathrm{kDa}$. Outre de puissants inhibiteurs plasmatiques comme l' $\alpha_{2}$-macroglobuline, des inhibiteurs tissulaires appelés TIMP (tissue inhibitors of matrix metalloproteinases) inhibent spécifiquement les MMP en se couplant à leur site catalytique. La dégradation de la matrice extracellulaire par les MMP est donc finement réglée à plusieurs niveaux successifs : leur expression et leur sécrétion, leur activation et leur inhibition [14].

Si les analogies de structure moléculaire des MMP indiquent qu'elles dérivent toutes d'un ancêtre commun $[14,15]$, leurs activités enzyma- 


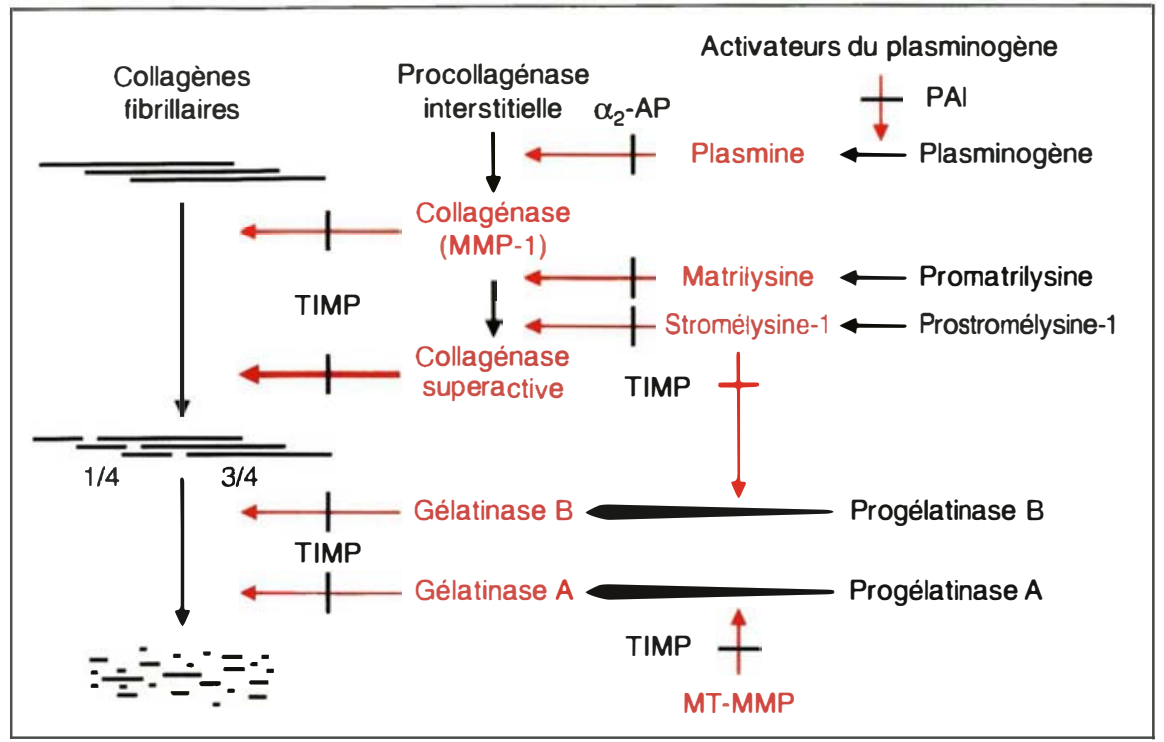

tiques permettent de distinguer au moins trois sous-groupes: les collagénases, les gélatinases et les stromélysines. Ia collagénase interstitielle (MMP-1), la collagénase de neutrophile (MMP-8) et la collagénase-3 [16] sont les seules enzymes capables de cliver, à pH neutre, la triple hélice des collagènes fibrillaires de types I, II et Ill, exclusivement entre les acides aminés 775 et 776 (glycine-leucine ou -isoleucine), produisant les fragments caractéristiques $3 / 4$ et $1 / 4$ de la molécule initiale. Ces fragments peuvent ensuite être dégradés par les gélatinases A (MMP-2) et B (MMP-9), par ailleurs capables d'hydrolyser les collagènes de types IV, V, VII et X. Les stromélysines-1 (MMP-3) et -2 (MMP-10), et la matrilysine (MMP-7) dégradent diverses protéines de la matrice extracellulaire, notamment le collagène de type IV, des protéoglycannes et des glycoprotéines telles que la fibronectine et la laminine. Enfin, quelques MMP récemment découvertes ne sont pas regroupées avec les précédentes: la stromélysine-3 (MMP-11), dont on ne connaît pas encore le ou les substrats naturels, la métalloélastase de macrophage et la métalloprotéinase de type membranaire [13] $\left(\mathrm{m} / \mathrm{s} n^{\circ} 10\right.$, vol. 10, p. 1(66)), laquelle est impliquée dans l'activation de la progélatinase $\mathrm{A}$.

De nombreuses protéases sont capables d'activer les procollagénases in vitro, mais le ou les activateurs phy- siologiques sont encore inconnus. La stromélysine-1, la stromélysine-2 et la matrilysine superactivent la collagénase interstitielle [12] mais elles doivent elles-mêmes être activées et leur action sur la procollagénase est particulièrement lente, sauf après une protéolyse préliminaire de la procollagénase par un autre activateur. Dans l'endomètre, les enzymes les plus susceptibles de jouer un rôle déterminant dans l'activation de la procollagénase interstitielle sont la kallikréine, la cathepsine B et la plasmine [17].

La plasmine est une protéase à large spectre d'action impliquée dans le remodelage de la matrice extracellulaire dans de nombreux processus physiologiques et pathologiques [18]. Elle est particulièrement active dans l'endomètre, surtout en période périmenstruelle [19] durant laquelle elle dégrade la fibrine des thrombi intravasculaires, ce qui rend le sang menstruel incoagulable. Les deux types d'activateurs du plasminogène, le type tissulaire et le type urokinase, ainsi que leurs inhibiteurs spécifiques, sont produits par l'endomètre [20]. L'activateur tissulaire du plasminogène se fixe préférentiellement sur la fibrine et agirait donc essentiellement dans la destruction des thrombi intravasculaires. L'urokinase, quant à elle, se lie à des récepteurs cellulaires de forte affinité [21] et jouerait un rôle dans le remodelage
Figure 2. Rôle et régulation des métalloprotéinases matricielles (MMP) dans la dégradation extracellulaire des collagènes fibrillaires. Le schéma est une simplification du mode d'action supposé physiologique de certaines MMP, de leurs mécanismes d'activation en cascade par d'autres protéases, et du contrôle de leur activation et de leur action par des inhibiteurs. Le modèle proposé n'intéresse que la dégradation des collagènes fibrillaires formant l'armature conjonctive des tissus. $L^{\prime} \alpha_{2}$-macroglobuline, inhibiteur de toutes les protéases mentionnées, n'est pas représentée. $\alpha_{2}-A P, \alpha_{2}$-antiplasmine; $M T-M M P$, métalloprotéinase matricielle de type membranaire; PAl, inhibiteurs des activateurs du plasminogène; TIMP, inhibiteurs tissulaires des MMP.

de la matrice extracellulaire en activant le plasminogène de manière focale, à la surface des cellules. Les macromolécules constitutives de la matrice extracellulaire seraient alors dégradées à la suite de l'activation de métalloprotéinases matricielles ou, pour certaines d'entre elles, directement par la plasmine.

La figure 2 schématise certains aspects du rôle et des interactions de ces protéases dans la lyse de la matrice extracellulaire.

\section{Les métalloprotéinases matricielles dans l'endomètre}

La plupart des métalloprotéinases matricielles ont été détectées dans l'endomètre, mais leur expression durant le cycle menstruel est variable. La collagénase interstitielle n'est exprimée que pendant la période périmenstruelle. En effet, son ARN messager n'est décelé par Northern blot qu'à ce moment du cycle (figure 3) [22, 23]. La collagénase interstitielle et son ARN messager sont alors localisés de manière focale dans des cellules stromales [24, 25], notamment au voisinage et dans la paroi d'artérioles spiralées [25]. De même, les ARN messagers de la stromélysine-1, de la stromélysine- 2 et de la gélatinase $B$ ne sont transcrits que pendant la période périmenstruelle $[23,24]$, durant laquelle ils sont 


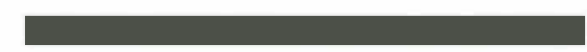

RÉFÉRENCES

25. Kokorine I, Pladys A, Marbaix E, Okada Y, Henriet P, Donnez J, Eeckhout Y, Courtoy PJ. Cellular origin of interstitial collagenase in human endometrium. Proceedings of the 14th meeting of the Federation of European the 14 th meeting of the Federation of European
Connective Tissue Societies, Lyon, 1994; abstract D10.

26. Rodgers WH, Osteen KG, Matrisian LM. Expression and localization of matrilysin, a matrix metalloproteinase, in human endometrium during the reproductive cycle. $A m$ J Gynecol Obstet 1993; 168: 253-60.

27. Marbaix E, Donnez J, Courtoy PJ, Eeckhout Y. Progesterone regulates the activity of collagenase and related gelatinases $A$ and $\mathrm{B}$ in human endometrial explants. Proc Natl Acad Sci USA 1992; 89: 11789-93.

28. Martelli $M$, Campana A, Bischof $\mathbf{P}$ Secretion of matrix metalloproteinases by human endometrial cells in vitro. I Reprod Fertil 1993; 98 : 67-76.

29. Rawdanowicz TJ, Hampton AL, Nagase H, Woolley DE, Salamonsen LA. Matrix metalloproteinase production by cultured human endometrial stromal cells: identification of interstitial collagenase, gelatinase$A$, gelatinase-B, and stromelysin- 1 and their differential regulation by interleukin-1 $\mathrm{a}$ and tumor necrosis factor- $\alpha$. J Clin Endocrinol Metab 1994; 79 : 530-6.

30. Schatz F, Papp C, Toth-Pal E, Lockwood CJ. Ovarian steroid-modulated stromelysin-1 expression in human endometrial stroma and decidual cells. I Clin Endocrinol Metab $1994 ; 78: 1467-72$

31. Casslén B, Anderson A, Nilsson IM, Astedt B. Hormonal regulation of the release of plasminogen activators and of a speci fic activator inhibitor from endometrial tissue in culture. Proc Soc Exp Biol Med 1986; 182: 419-24.

32. Marbaix E, Cornillie FJ, Donnez J, Eeckhout Y, Courtoy PJ. Applications de la culture organotypique de l'endomètre humain: perspectives nouvelles dans l'évaluation des modifications morphologiques de l'endomètre humain. Contraception Fertilité Sexualité $1990 ; 18: 895-9$.

33. Hourihan HM, Sheppard BL, Bonnar J. The morphologic characteristics of menstrual hemostasis in patients with unexplained menorrhagia. Int I Gynecol Pathol 1989; 8: 221-9.

34. Alexander NJ, D'Arcangues C. Steroid hormones and uterine bleeding. Washington:

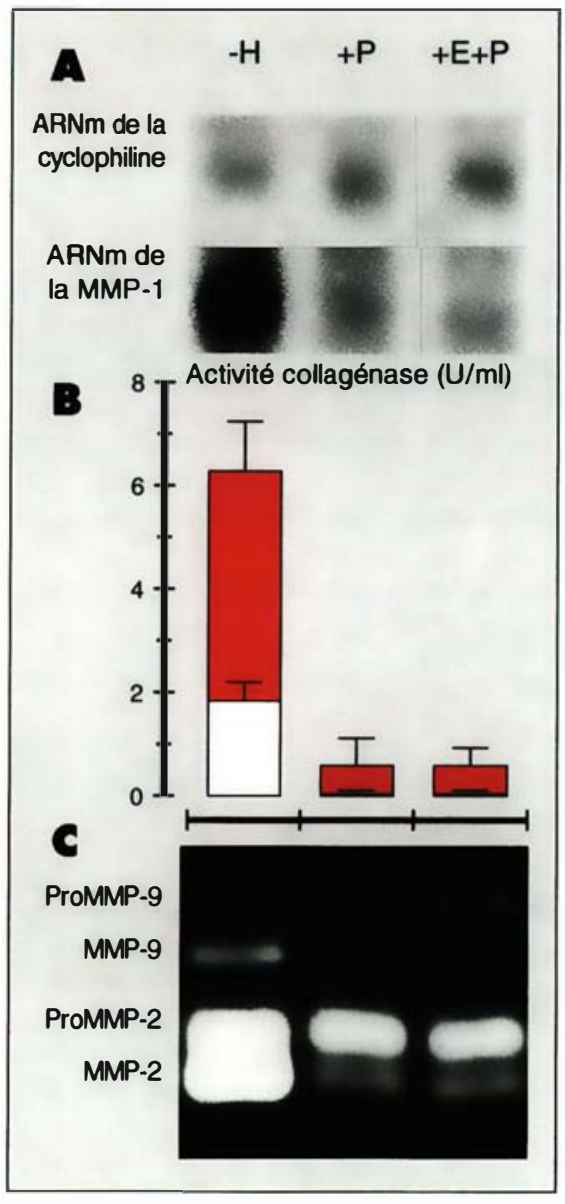

détectés de manière focale dans des cellules stromales. En revanche, l'ARN messager de la gélatinase $A$ est détecté dans l'ensemble des cellules stromales de l'endomètre et tout au long du cycle menstruel, quoiqu'en plus grande quantité en fin de phase sécrétoire [24]. Enfin, la matrilysine est exprimée non seulement pendant la période périmenstruelle mais aussi pendant la phase proliférative. En outre, au contraire des autres métalloprotéinases matricielles, la matrilysine et son messager sont localisés dans l'épithélium glandulaire et non dans les cellules stromales [26].

La régulation des métalloprotéinases matricielles de l'endomètre a pu être mieux caractérisée dans différents systèmes de culture. Dans les conditions appropriées, des explants de muqueuse endométriale [22, 27] et des cultures primaires de cellules stromales de l'endomètre [28-30] sécrètent la collagénase interstitielle, les gélatinases A et B et la stromélysine-1. Ces protéases ont été identifiées dans le milieu de culture par dosage enzymatique [27, 29], par Western blot $[22,29,30]$ et par zymographie, technique révélant leur activité après séparation en électrophorèse dans une matrice où leur substrat (gélati-

Figure 3. Régulation hormonale de l'expression de collagénase et de la sécrétion de gélatinases par les explants d'endomètre. Des explants d'un endomètre prélevé en fin de phase sécrétoire ont été cultivés pendant deux jours sans hormone sexuelle (-H), en présence de progestérone $100 \mathrm{nM}(+P)$ ou d'cstradiol $1 \mathrm{nM}$ et de progestérone $100 \mathrm{nM}(+E+P)$. Dans cette expérience, l'ajout d'œstradiol n'a pas accentué l'effet inhibiteur déjà très marqué de la progestérone seule. A. Northern blot. Au terme de la culture, I'ARN total $(3 \mathrm{\mu g})$ extrait des explants a été analysé par Northern blot et hybridé avec une sonde de l'ARNm de la collagénase interstitielle (MMP-1; 2,2 kb). Après décapage de cette sonde, la membrane a été réhybridée avec une sonde de l'ARNm de la cyclophiline $(1,0 \mathrm{~kb})$, utilisé ici comme contrôle de la quantité d'ARNm déposée dans chaque piste, car son expression n'est pas réglée par les hormones sexuelles. B. Dosage enzymatique. L'activité collagénase sécrétée au cours du deuxième jour de culture a été mesurée dans les milieux conditionnés, soit sans traitement préalable (colonne blanche) de manière à apprécier l'activité spontanée de la collagénase, soit après traitement par l'acétate aminophénylmercurique (colonnes roses) pour déterminer l'activité collagénase totale (active + latente). Chaque colonne correspond à la moyenne de 3 ensembles de milieux conditionnés et les barres verticales représentent les écarts-types. C. Zymographie. Les pro-enzymes et les formes spontanément actives des gélatinases (ProMMP-9 et MMP-9; ProMMP-2 et MMP-2) sécrétées pendant le deuxième jour de culture ont été analysées par zymographie, en copolymérisant de la gélatine à l'acrylamide du gel d'électrophorèse. Les pro-enzymes sont artificiellement activées de manière irréversible par le dodécylsulfate de sodium utilisé lors de la migration électrophorétique. L'activité de chaque (pro-lenzyme peut être estimée par la surface et l'intensité de décoloration du gel, correspondant à la dégradation du substrat. 


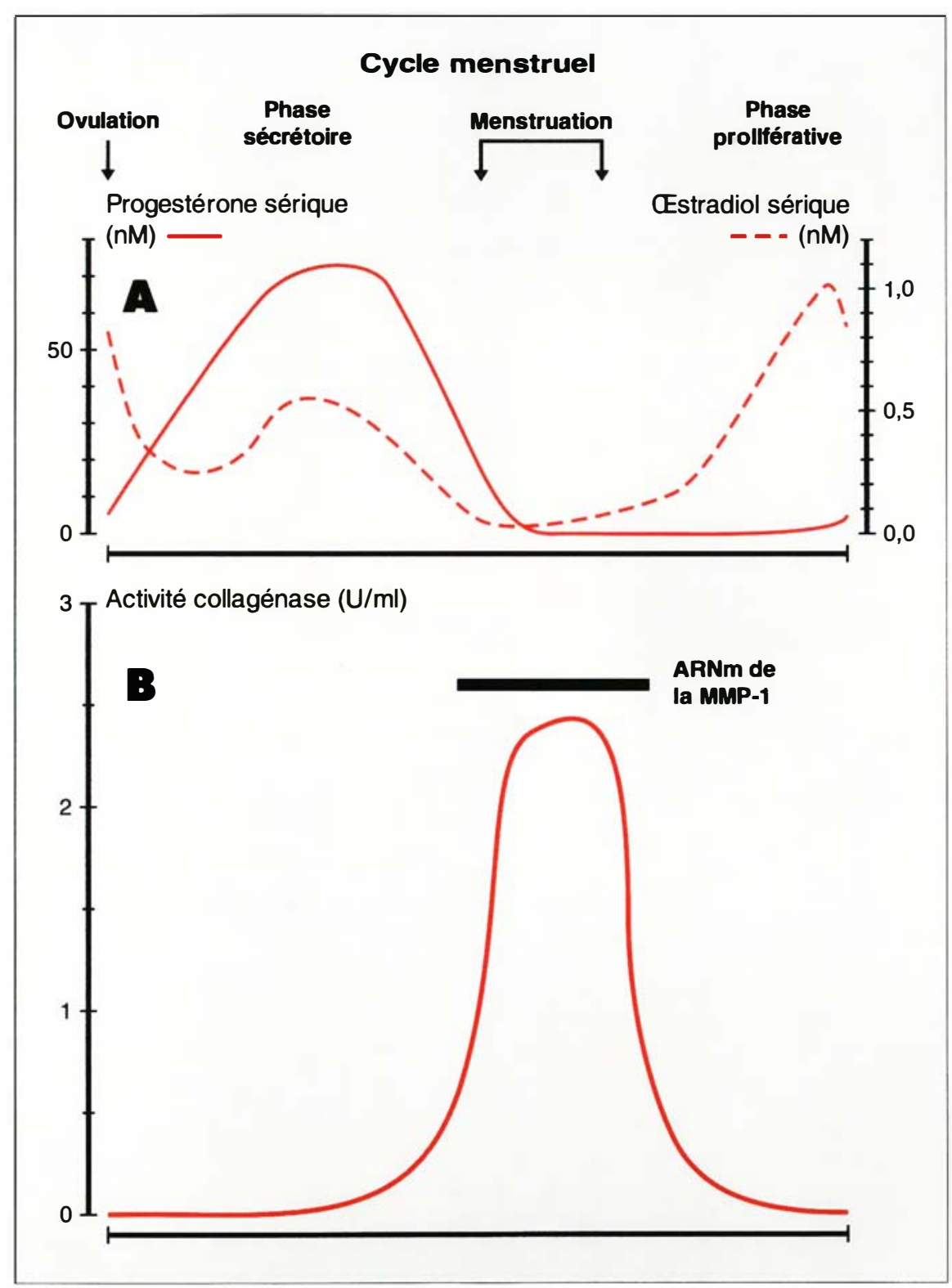

Figure 4 . Expression de la collagénase interstitielle au cours du cycle menstruel. A. Concentrations plasmatiques en progestérone (ligne continue) et en œstradiol (ligne discontinue) au cours du cycle menstruel, débutant ici par l'ovulation. B. Activité collagénase sécrétée pendant les 24 premières heures de culture sans hormone sexuelle par des explants d'endomètre prélevés au long du cycle menstruel. Les milieux conditionnés ont été traités à l'aide $d^{\prime}$ acétate aminophénylmercurique pour activer la forme latente de la collagénase, ce qui permet de mesurer la collagénase totale. La barre épaisse indique la période du cycle où l'ARN messager de la collagénase interstitielle est décelé dans le tissu frais par Northern blot. (D'après [22].) ne ou caséine) est copolymérisé à l'acrylamide [27-29]. Les explants d'endomètre sécrètent également les TIMP-1 et -2 [27].

L'ARN messager de la collagénase interstitielle est transcrit dans les explants d'endomètre en culture sans hormone, et la progestérone, surtout en présence d'œstradiol, en diminue fortement la concentration (figure 3) [22]. Cet ARN messager est détecté par hybridation in situ dans le cytoplasme de nombreuses cellules stromales d'explants d'endomètre. La protéine peut être immunolocalisée dans les mêmes cellules lorsque les explants sont cultivés en présence de monensine, utilisée ici pour retenir la protéine dans les cellules productrices et augmenter ainsi la sensibilité de sa détection [25].

En dehors de la période périmenstruelle, des explants de muqueuse endométriale ne sécrètent pratiquement pas d'activité collagénase au cours du premier jour de culture sans hormone sexuelle, mais cette activité apparaît durant les jours suivants où elle atteint un niveau considérable (figure 4 et résultats non présentés). En revanche, les explants d'endomètre prélevé en période périmenstruelle sécrètent, dès le premier jour de culture, une activité collagénase élevée. Nous avons montré que la majeure partie de cette activité ne peut être attribuée à la sécrétion de collagénase par les polymorphonucléaires neutrophiles, nombreux dans le tissu à ce moment du cycle [22].

La sécrétion d'activité collagénase par des explants d'endomètre est contrôlée par les stéroïdes sexuels (figure 3). En effet, la progestérone ajoutée à des concentrations physiologiques $(10-100 \mathrm{nM})$ inhibe fortement la sécrétion de collagénase pendant plusieurs jours de culture, sauf dans les endomètres menstruels qui n'expriment plus guère le récepteur de la progestérone [22]. L'œstradiol, à la concentration physiologique de $1 \mathrm{nM}$, exerce un effet inhibiteur moindre sur la sécrétion d'activité collagénase lorsqu'il est utilisé seul, mais potentialise nettement l'effet inhibiteur de la progestérone lorsque les deux stéroĩdes sont combinés [22]. L'induction du récepteur de la progestérone par l'œstradiol pourrait expliquer ces résultats. La progesté- 
rone inhibe également la sécrétion et l'activation de la gélatinase $A$ et surtout de la gélatinase $B$ dans l'endomètre en culture organotypique (figure 3) [27], ainsi que l'expression de la stromélysine-l dans des cultures de cellules stromales de l'endomètre [30].

Si l'expression et la sécrétion des MMP sont déréprimées juste avant et pendant la menstruation à la suite de la chute de la concentration des stéroïdes sexuels, encore faut-il que ces MMP soient activées durant cette période pour y jouer un rôle. L'activité collagénase sécrétée par un endomètre non périmenstruel après quelques jours de culture sans hormone sexuelle reste essentiellement sous forme latente. Au contraire, une proportion significative de la collagénase sécrétée lors du premier jour de culture d'un endomètre menstruel est détectée sous une forme spontanément active [22]. Parmi les activateurs potentiels de la collagénase interstitielle, l'urokinase subit une régulation hormonale appropriée pour exercer cette fonction. En effet, la progestérone inhibe la production d'urokinase par des explants d'endomètre en culture et stimule l'expression d'un de ses inhibiteurs [31]. Il en résulte que l'activité du système des activateurs du plasminogène est maximale en période périmenstruelle [19].

\section{Conclusions et perspectives}

La restriction à la période périmenstruelle de l'expression de la collagénase interstitielle, de la gélatinase $B$ et de la stromélysine-l et le caractère focal de leur expression, de même que le contrôle serré de l'expression et de l'activation de la collagénase interstitielle et de la gélatinase B par la progestérone, suggèrent fortement que ces enzymes interviennent dans la dégradation de la matrice extracellulaire de l'endomètre. Cela rendrait compte du collapsus focal du stroma observé pendant la période périmenstruelle, conduisant à l'élimination de fragments de muqueuse au cours de la menstruation.

La présence des enzymes susceptibles de dégrader le substrat adéquat à l'endroit et au moment appropriés
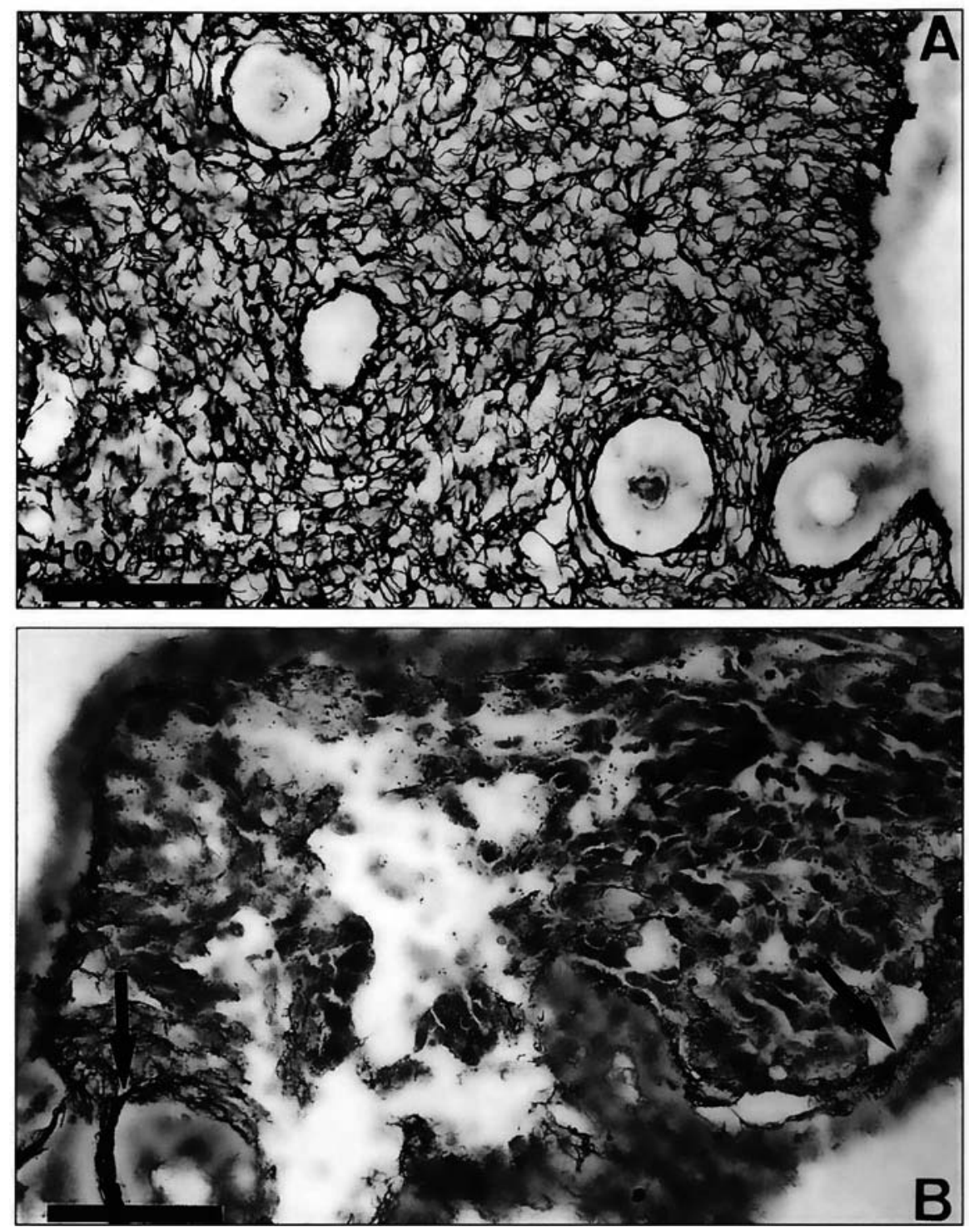

Figure 5. Lyse spontanée de la matrice extracellulaire de l'endomètre en culture. Des explants d'un endomètre prélevé en phase proliférative ont été cultivés pendant deux jours en présence d'cstradiol $1 \mathrm{nM}$ et de progestérone $100 \mathrm{nM}$ (A) ou sans hormone sexuelle (B). Au terme de la culture, les explants ont été fixés, enrobés de paraffine, et des sections histologiques ont été réalisées et colorées à l'argent. Le réseau dense de fibres réticuliniques observé dans l'explant cultivé en présence des stéroïdes sexuels est comparable à celui observé dans les endomètres frais en dehors de la menstruation (voir figure 1B et D), tandis que la disparition complète de ces fibres dans l'explant cultivé sans hormone, sauf par endroits sous l'épithélium de surface et autour d'une glande (flèches), rappelle l'aspect d'un endomètre menstruel (voir figure 1F). Les barres correspondent à $100 \mu \mathrm{m}$.

tion effective dans les mécanismes de la menstruation. Cette hypothèse demande confirmation par l'utilisation d'inhibiteurs spécifiques, un type d'expérimentation pour lequel le système de culture organotypique de l'endomètre humain est particu- lièrement approprié [32]. En effet, des explants d'endomètre prélevés en dehors des menstrues et cultivés sans hormone sexuelle pendant quelques jours se rétractent et présentent une désorganisation de leur trame réticulinique comparable à cel- 
le observée dans l'endomètre menstruel (figure 5). Au contraire, la matrice extracellulaire est bien préservée dans d'autres explants du même tissu cultivés en présence d'œstradiol et de progestérone.

Si d'autres perturbations, notamment des lésions de l'endothélium vasculaire [33], participent aussi aux hémorragies menstruelles, la rétraction stromale résultant de la lyse de la matrice extracellulaire est une modification constante de la menstruation. La rétraction du stroma endométrial est d'ailleurs observée au cours du cycle reproducteur d'autres mammifères non primates. Une meilleure compréhension du déclenchement et du contrôle de la lyse de la matrice extracellulaire devrait ouvrir de nouvelles perspectives dans le traitement des ménorragies et métrorragies, souvent sans cause anatomique. Elle pourrait aussi conduire à un meilleur contrôle des pertes de sang légères occasionnées par un traitement contraceptif hormonal, qui sont souvent responsables de l'interruption de la contraception et font ainsi obstacle au contrôle de la démographie mondiale [34]

\section{Remerciements}

Les auteurs remercient le Fonds National de la Recherche Scientifique et les Services fédéraux des affaires scientifiques, techniques et culturelles de Belgique, ainsi que Ipsen-Biotech, France, et M.E. Bertrand pour leur soutien. Ils sont également reconnaissants aux professeurs $H$. Nagase, Kansas City, USA, et Y. Okada, Kanazawa, Japon, ainsi qu'à Fuji Chemical Industries Ltd, Japon, pour leurs dons généreux de sondes et d'anticorps spécifiques. Enfin, ils expriment leur gratitude à M.Ch. Balden, A. Herssens-Marcelis, P. Lefebvre-Lemoine et F. N'Kuli pour leur aide technique, à $\mathrm{S}$. Lagasse pour le travail photographique et à Y. Marchand pour la recherche bibliographique.

\section{Summary}

Collagenase and related matrix metalloproteinases : a prominent role in the initiation of menstruation?

The human endometrium shows striking structural changes during the menstrual cycle. If no pregnancy develops, the fall of plasma progesterone and estradiol induces extracellular matrix degradation leading to haemorrhagia and mucosal shedding, i. e. menstruation. The mechanisms of endometrial tissue breakdown remain obscure. A major role of lysosomal hydrolases is not supported by biochemical evidence, whereas recent studies by several laboratories including our own suggest that interstitial collagenase and related matrix metalloproteinases (MMPs) are involved. We have demonstrated that human endometrium in tissue culture can produce interstitial collagenase and gelatinases $\mathrm{A}$ and B. Both the expression and the activation of these MMPs were inhibited by physiological concentrations of progesterone and estradiol. Moreover, in fresh human endometrial tissue, expression of mRNA, protein and activity of interstitial collagenase, as well as of mRNA of several other MMPs is limited to the perimenstrual period. In such tissues, immunolocalisation and in situ hybridization show that the expression is focal. The tight hormonal control as well as the restricted temporal and spatial expression of MMPs in human endometrium all point to a pivotal role of interstitial collagenase and other MMPs in the initiation of menstruation.

\section{TIRÉS À PART}

